\title{
Uncertainties in a carbon footprint model for detergents; quantifying the confidence in a comparative result
}

\author{
Arjan de Koning • Diederik Schowanek • \\ Joost Dewaele • Annie Weisbrod • Jeroen Guinée
}

Received: 16 February 2009 / Accepted: 17 June 2009 /Published online: 29 October 2009

(C) The Author(s) 2009. This article is published with open access at Springerlink.com

\begin{abstract}
Background, aim, and scope A new trend driven by climate change concerns is the interest to label consumer products with a carbon footprint (CF) number. Here, we present a study that examines the uncertainty in the estimated CFs of a liquid and a compact powder detergent and how the uncertainty varies with the type of comparison one wishes to make.

Materials and methods A simplified CF model for detergents, encompassing all life cycle stages, has been used for the calculation of CFs. The CFs for the two detergents were compared under three different cases: (1) a situation where most of life cycle assessment (LCA) system is similar, (2) a situation where the LCA background systems may be different but certain choices with regard to system boundaries are standardized, and (3) a situation where the LCA background system, choices of system boundaries,
\end{abstract}

Responsible editor: Matthias Finkbeiner

Electronic supplementary material The online version of this article (doi:10.1007/s11367-009-0123-3) contains supplementary material, which is available to authorized users.

A. de Koning $(\bowtie) \cdot J$. Guinée

Institute of Environmental Sciences (CML), Universiteit Leiden, P.O. Box 9518, 2300 RA Leiden, The Netherlands

e-mail: koning@cml.leidenuniv.nl

D. Schowanek $\cdot$ J. Dewaele

Procter \& Gamble Eurocor,

Temselaan 100,

1853 Strombeek-Bever, Belgium

\section{A. Weisbrod}

Procter \& Gamble Company,

Winton Hill Business Center, 6280 Center Hill Avenue,

Cincinnati, OH 45224, USA and foreground system may also be different. Uncertainty in the CFs was calculated for each of the three comparison situations using a stepwise sensitivity/uncertainty analysis approach.

Results The stepwise approach makes it possible to obtain reliable uncertainty estimates without the need to have very good uncertainty descriptions for every input parameter. Only a few input parameters were found to drive the uncertainty of the CF values. For case 1, the uncertainties in the difference between the CF of the ultraliquid and compact powder products are very small. The CF of compact powder is always larger than that of the ultraliquid product. In case 3, the uncertainties become much larger, such that in $23 \%$ of the cases, a CF comparison would wrongly indicate that the compact powder product has a lower CF than the ultraliquid product. Case 2 falls between the extremes of cases 1 and 3 .

Discussion One of the challenges of developing userfriendly CF methods based on the ISO 14040 framework is to ensure a high level of comparability of CF values, such that misleading or oversimplified conclusions can be avoided. Our analysis shows how the uncertainty margins around the calculation of a CF for a set of given products will broaden as the assessment moves from an "internal" comparison to a comparison with data from third parties where there is no specific information how these data have been obtained. CF calculations based on internal comparisons can lead to very clear distinctions between products and illustrate the utility of a $\mathrm{CF}$ tool to optimize the environmental performance of products using difference analysis.

Conclusions CF calculations for products can only provide a fair comparison if the LCA background system used for the two products is the same and exactly the same choices in the foreground system are made. In practice, this would 
require consultation and agreement on specific product category rules.

Recommendations and outlook Simplification is needed for a wider adoption of uncertainty analysis in CF and LCA. This article introduces some first steps towards such simplification, but more work is needed both on the theoretical and practical aspects of simplified uncertainty analyses.

Keywords Carbon footprint · Detergents · FAST method . Monte Carlo analysis · Sensitivity analysis .

Uncertainty analysis

\section{Background, aim, and scope}

A recent United Nations Environment Program (2007) report assessed growing impacts on the environment over the last 20 years. Climate change was identified as one of the most pressing problems, although the condition of freshwater supplies, agricultural land, and biodiversity were considered to be of equal concern. Media has focused heavily on the growing concern for climate change, which has resonated in different layers of society around the world. As a result, climate issues are increasingly part of today's political agenda. While energy (and hence carbon) saving traditionally found a place in industrial "ecoefficiency" and cost-saving initiatives, climate change is now regarded as an environmental responsibility of the entire society, including governments, industries, and individual consumers.

Environmental impacts related to products or services are best examined using a life-cycle-based method. One environmental impact is the cumulative amount of greenhouse gasses (GHGs) emitted by a product or service throughout its life cycle. This cumulative amount of GHGs, expressed in kilogram $\mathrm{CO}_{2}$ equivalents released to the atmosphere, represents the carbon footprint (CF) of a product or service.

A new trend is that some retailers have started labeling their own brands of consumer products with a carbon label (CL) and have been asking their suppliers for the CF scores of their respective products. The objective is to enable shoppers to compare one product to another on a CF basis. Unfortunately, these early developments have taken place in the absence of a fully harmonized methodology and therefore suffer from inconsistencies in approach and quality differences between the underlying LCAs. For example, differences between methodologies have become apparent with respect to the GHGs included $\left(\mathrm{CO}_{2}\right.$ only, versus all potential GHG emissions), system boundaries (Matthews et al. 2008; Johnson 2008), and allocation (Guinée and Heijungs 2007).
To address these problems, a number of separate initiatives have been started to develop harmonized methodologies for carbon footprinting of consumer products and services within an LCA framework. An important example is the PAS2050 specification (BSI 2008) developed in the UK by the Carbon Trust, DEFRA, and BSI. PAS 2050 builds on existing LCA methods established through ISO 14040 and ISO 14044 by specifying requirements for the assessment of the life cycle GHG emissions of goods and services. Similar initiatives are under development by ISO (2007) and WBSCD/WRI (2007).

As an LCA where the analysis is limited to GHGs, a CF calculation has a number of inherent complexities that are not fully recognized (Pant et al. 2008). CFs, like any other output of a LCA model, are inherently uncertain because an LCA model contains many uncertain input parameters. This paper deals with the essential question "when is the CF of product A significantly different from that of alternative Product B?" This issue is important for internal company decision making (e.g., to guide product development) but probably even more so when comparing the CFs of product alternatives in commerce. Single-number product carbon labels may provide a false sense of accuracy to the user. This may not only be subject to scientific criticism but can also be legally challenged, since a CF can be regarded as an environmental claim, and some CF schemes constitute the basis of subsidy granting systems (Staatsblad 2006; US 1998).

In this paper, we will present the results of an uncertainty analysis on the calculated CFs of two different types of laundry detergents that fulfill the same function and associated implications for CL schemes. We will describe how the uncertainties highly depend on the way the products are compared. Defining different comparison situations technically means that input parameters for the CF models are correlated differently. Two different approaches are used that deal with correlations between input parameters of CF models. Also, an approach for a stepwise sensitivity and uncertainty analysis is presented, which enables users to obtain reliable uncertainty estimates without the need to have very precise uncertainty descriptions for every input parameter. The study is restricted to the analysis of uncertainties in the life cycle inventory (LCI) calculation. Uncertainties in the life cycle impact assessment are not addressed.

\section{Materials and methods}

\subsection{Specifying uncertainty}

The uncertainty in life cycle models can be categorized in many different ways (Heijungs and Huijbregts 2004). 
Following the often employed categorization by the USEPA (1989) which is also helpful in the context of CF models, three types of uncertainty can be distinguished in LCA models:

- Parameter uncertainty: uncertainty in observed or measured values used as input to a model

- Model uncertainty: uncertainty about the way the interaction between technosphere and biosphere has to be modeled leads to uncertainty

- Scenario uncertainty: relates to choices made in constructing scenarios. Typical choices include choice of impact assessment method and allocation

Depending on the specific LCA model of interest, any of the three types of uncertainty can have the largest contribution to the final result of the LCA model (Lloyd and Ries 2007). Therefore, a comprehensive uncertainty analysis cannot exclude a priori any of the three types of uncertainties. Scenario and model uncertainties are not commonly addressed in LCA uncertainty studies. Of all LCA studies examined by (Lloyd and Ries 2007), only $29 \%$ considered these sources of uncertainty and often only in the life cycle impact assessment.

Introducing and analyzing parameter uncertainty in LCA is an established practice (Lloyd and Ries 2007). Samplingbased methods and analytical methods are available to address this kind of stochastic uncertainty. Parameter uncertainty is nowadays also systematically addressed in the ecoinvent $v 1.3^{1}$ database where every process description is accompanied by a systematic uncertainty assessment (Frischknecht et al. 2007).

For the detergent CF model under consideration, it is the LCI phase where model and scenario uncertainty is to be addressed. However, a full systematic analysis of the contribution of scenario and model uncertainty in LCA is not well developed, and no guidelines exist for doing so. Therefore, we developed our own approach and methods. To assess the influence of model and scenario uncertainty on the calculated CF model, alternative scenarios and models should be studied. It very much depends on expert judgement which alternative scenarios are selected as plausible alternatives. Scenarios can be developed in different ways:

1. Changing one unit process for another unit process having different values for the inputs and outputs (e.g., as a result of different allocation method, or different wash temperature)

2. Removing a unit process. This would be equal to changing the system boundaries of the study

\footnotetext{
${ }^{1}$ The ecoinvent 2.0 database was not used in our study because it does not include the uncertainty for the aggregated processes calculated from the uncertainty in the unit processes.
}

3. Changing the variability assigned to the value of the inputs and outputs of the unit process

As will be shown later in the selection of scenarios to be studied, all three cases are of relevance. Options 1 and 2 are clear as they are, but option 3 for the introduction of scenarios needs some further discussion. While the definition of parameter and scenario uncertainty seems to indicate that these types of uncertainties can be clearly distinguished, in practice they cannot. For instance, the simplified $\mathrm{CF}$ model contains many aggregated processes and their related $\mathrm{CF}$ (e.g., $\mathrm{CF}$ related to paper and cardboard production). The $\mathrm{CF}$ of these aggregated processes is the calculated result of a cradle-to-gate life cycle analysis, containing all the three uncertainty types as discussed above. However, the three types of uncertainty can only manifest themselves by contributing to the uncertainty of the final result of the cradle-to-gate LCA, i.e., the uncertainty of the result for the $\mathrm{CF}$ of the aggregated process. Thus, the uncertainty in the aggregated process can only be expressed as uncertainty in the parameter value, although it actually is the aggregate of model, scenario, and parameter uncertainty. Therefore, incorporating scenario and model uncertainty in the uncertainty analysis can be done in the same sampling-based framework as parameter uncertainty. The samples consist of different combinations of different scenarios with a subjective probability reflecting the preference of the decision maker for an alternative (Efron and Tibshirani 1991).

\subsection{Comparison situation 1}

A first comparison situation deals with a case where the majority of the processes in the life cycle of the two compared products is identical (which we will denote as comparison situation 1 or an "intrapractitioner" comparison (IPC)). In such a situation, an LCA practitioner uses a single set of input (background) data to calculate the cradleto-grave emissions of GHGs for different products. A typical example is when a company's internal expert compares two product alternatives for the same function, which are relatively similar in overall design and composition using a single LCA background dataset. That expert has full control over the design of the study and data selection.

In principle, all uncertainties in the economic flows and environmental interventions should be taken into account when comparing products in comparison situation 1 . However, values for the shared economic flows and environmental interventions will be fully correlated in an IPC situation. This means for instance that the same selected process in the background in the LCA of product 1 is also used in the background system of product 2 . The 
result of having many fully correlated input parameters is that the uncertainties calculated for the product $\mathrm{CFs}$ are not independent from each other. A low value for the $\mathrm{CF}$ for product 1 will likely coincide with a low value for product 2 .

From a methodological perspective, we can use two methods to account for the correlation of common processes in the LCA or CF of the two products:

1. The analytical approach. Perform a separate uncertainty analysis for products 1 and 2 using fully correlated input parameters for the common processes in the LCA's of products 1 and 2. Compare the products, taking into account that the $\mathrm{CF}$ for both products are highly correlated.

2. The subjective approach. Because the LCA practitioner chooses to include the emissions for a process according a specific model, it becomes a subjective choice, just like the LCA practitioner can, e.g., decide upon the use of a certain allocation scenario. Because the LCA practitioner is fully "in control" of which input data to use for a process and thus defines the boundaries and assumptions about the significance of a particular process, no uncertainties remain with respect to the choice of the selected process. Therefore, we can disregard uncertainties in the processes common to the two products. The subjective approach can also be seen as a simplified method to take into account that the CFs of both products are correlated.

Efforts to standardize the LCA methodology can be seen as a subjective approach to reduce uncertainties in LCA results, making the results of LCAs comparably better. Little information with regard to the appropriateness of these perhaps complementary approaches exists. In this study, we will show by means of an example that the subjective approach can be justified in our case.

Actual uncertainties that were taken into account in our study for comparison situation 1 relate to the following six input parameter groups: amount and types of ingredients in the detergents, amounts and types of packaging material, emissions during formulation of the detergent, transport distance for the raw material and finished product, and the oxygen demand of the degradation of detergent ingredients in the wastewater treatment plant (WWTP). The uncertainties taken into account in the IPC analysis for these six input parameters groups only cover parameter uncertainty if these parameters describe a single (unaggregated) technical coefficient in a foreground process (e.g., the amount of packaging materials for detergent packaging). If an input parameter resembles an aggregated (cradle-to-gate) results (e.g., the GHG emissions during the formulation of the detergent), both parameter, model, and scenario uncertainties are addressed, as described previously.
The uncertainty in the input parameters that describe the characteristics of common processes is not included in the sensitivity and uncertainty analyses. See Table 1 for an overview of this comparison situation. A detailed specification of the uncertainty for all input parameters can be found in the "Electronic supplementary material."

\subsection{Comparison situation 2}

The second case for comparisons represents a situation where two independent LCA researchers follow ISO 14044 LCA standards plus a set of extra standardization rules. For example, Type III Environmental Labels or Declarations (cf ISO 14025; ISO 2000) are used by some product sectors, and these may have "product category rules" (PCRs). Although PCRs do not exist today for the detergent sector, a hypothetical example is that a PCR states that, for the comparison of the $\mathrm{CF}$ of detergents, the reference wash temperature shall be $40^{\circ} \mathrm{C}$; wash water has medium water hardness, and an energy class A washing machine shall be used. In effect, this means that the PCRs partly specify a common system on which to base the comparison of the detergent $\mathrm{CF}$, with the aim to improve consistency and repeatability. We will call this "comparison situation 2" or the "multipractitioner" comparison following PCR (MPC PCR).

For this comparison situation, uncertainties of all input parameters have to be taken into account, except when specific product category rules have been defined that prescribe the use of a common process. For this situation, such rules have only been defined for the use phase of the detergent. It has been (arbitrarily) decided to compare detergents using a fixed washing temperature of $40^{\circ} \mathrm{C}$ and to use a fixed $\mathrm{CF}$ for the electricity needed during washing of $570 \mathrm{~g} \mathrm{CO}_{2} \mathrm{eq} / \mathrm{kWh}$. The washing machine to be considered is an A-class energy-efficient washing machine. The energy efficiency of the washing machine used in the analysis was not defined so it was treated as uncertain.

The uncertainties taken into account in comparison situation 2 only cover parameter uncertainty if these parameters describe a single (unaggregated) technical coefficient in a foreground process. If an input parameter resembles an aggregated (cradle-to-gate) results, both parameter, model, and scenario uncertainty are addressed as described previously

\subsection{Comparison situation 3}

The third case of comparing two or more similar products arises when the $\mathrm{CF}$ of each product is calculated independently by different practitioners (which we will denote as comparison situation 3 or a multipractitioner comparison following ISO 14044 guidelines (MPC ISO 14044)). In this 


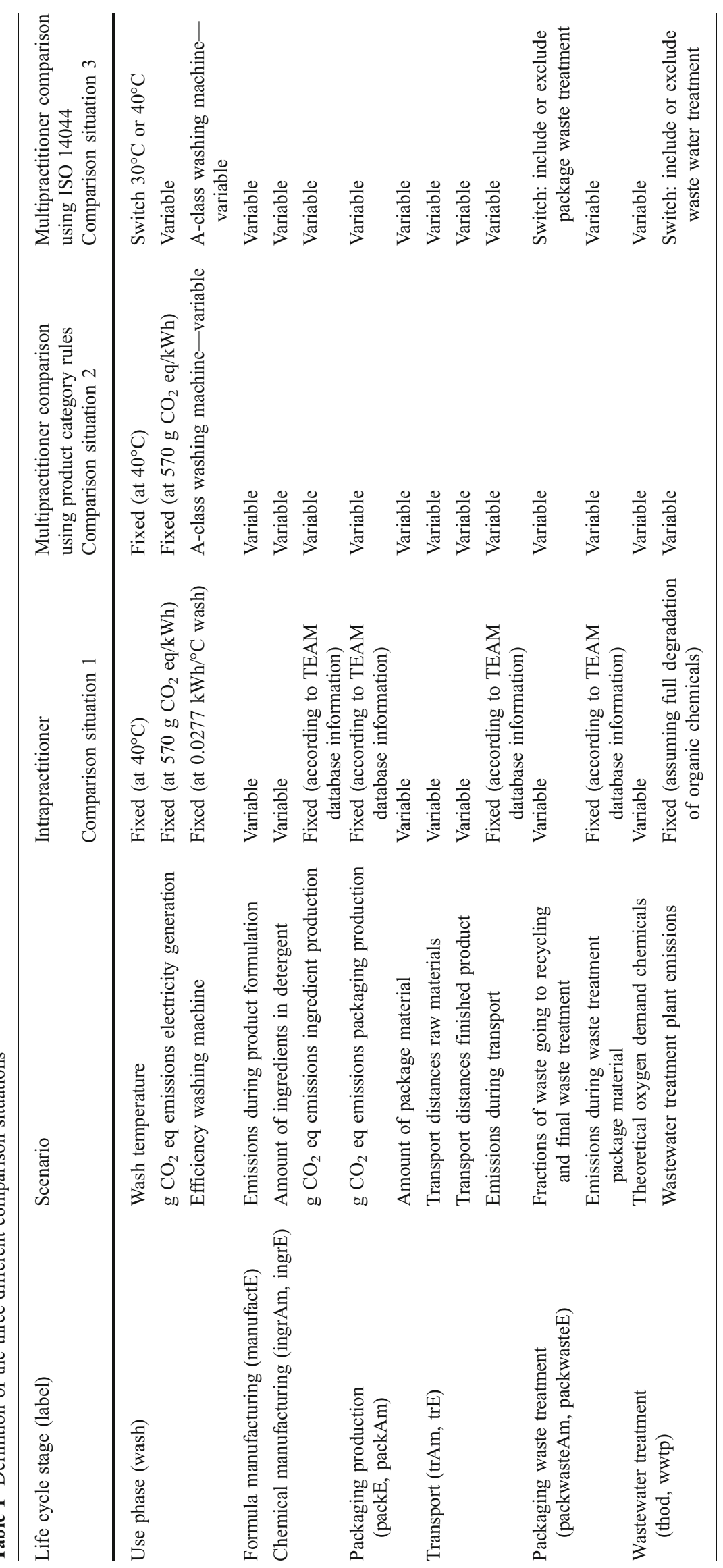


situation, one typically does not know if exactly the same processes have been used to estimate the CF of the products. In principle, the rules that define how the LCA has to be performed by each practitioner are laid down in ISO 14044 (ISO 2006a, b). However, ISO 14044 does not give a detailed protocol for specific products related to choice of system boundaries, allocation, selection of datasets, and use-phase parameters, and data unavailability can also influence whether a process is included. LCA practitioners working at different places will not know how the other's LCA or CF study was exactly done.

For this comparison situation, uncertainties of all input parameters have to be taken into account. Also, uncertainties related to the washing temperature and the incorporation of end-of-life stages of the product are now included in the analysis in contrast to comparison situation 2 where these were fixed choices, see Table 1 .

\subsection{Sources for uncertainty information}

Sources of information about uncertainty were the ecoinvent v1.3 database (Frischknecht et al. 2007), literature (Lloyd and Ries 2007; Huijbregts et al. 2003), and information available within P\&G. In this preliminary uncertainty specification, it was assumed that the uncertainty of all parameters could be described by a lognormal distribution function. Only in comparison situation 3 did we make use of a discrete distribution function for the uncertainty specification in the three discrete scenario choices. Besides the full correlation between the input parameters in comparison situation 1 that have been taken into account with the subjective approach, no further correlations were taken into account between the input parameters.

\subsection{Carbon footprint model for laundry detergents}

The CFs for the compact powder detergent and the ultraliquid detergent were calculated with a simplified LCA model for laundry detergents, implemented in Microsoft Excel. It is based on an existing LCA model for laundry detergents, which is the result of more than 10 years of development by P\&G and the detergent industry (Saouter and Van Hoof 2001). It calculates the CF per single wash, in this case in the UK, for a variety of laundry detergents, covering all life cycle stages. Even though this is a simplified model in which many aggregated processes have been specified, the model still contains about 180 input parameters. About $30 \%$ of these can be considered "primary data," i.e., they are specific to P\&G's manufacturing and distribution processes, while the remainder would be industry default values or related to the country energy grid. The life cycle stages included in the model are shown in Table 1. It should be remarked that few disposable consumer products today can rely on such a solid LCA "platform" for making comparative CF calculations.

The energy used during washing was modeled for a class A washing machine (NEF 2008). GHG emissions during electricity generation were derived from 2003 UK LCI data. GHG emissions during wastewater treatment are a combination of energy use for WWTP operations as well as the biodegradation of the laundry detergent ingredients. The global warming potentials (GWP) needed for the calculation of the CF from the GHG emissions are the GWPs with time horizon of 100 years published by the Intergovernmental Panel on Climate Change (Houghton et al. 2001). Uncertainties in the GWPs have not been taken into account in this study. GHG emissions during laundry detergent manufacturing have been taken from a number of published LCI data sources: surfactant LCI data have been taken from a large number of publications in the journal Tenside and Surfactants (EMPA 1999) for zeolites, carbonates and brighteners, and (Boustead and Fawer 1998) perborates. For chemicals not covered by the data sources above, ecoinvent v1.3 data (Frischknecht et al. 2007) were used to achieve $100 \%$ coverage of the detergent ingredients. GHG emissions during production of the package material have been taken from (APME 1996) and (BUWAL 1996). GHG emissions during formulation of the detergents were based on (Franke et al. 1995) for liquid and powder processing. Emissions during transport were estimated on the basis of emission data from (ETH 1996) and rough estimates of transport distances. Packaging waste treatment includes estimated recycling rates and amounts going into landfill and municipal solid waste incineration in the UK (UK Wisard model; Ecobilan 2008).

\subsection{Stepwise sensitivity and uncertainty analysis}

If the magnitude and influence of the different types of uncertainty were known a priori for all 180 input parameters, the assessment of the uncertainty in the CF would be straightforward. An uncertainty analysis could have been carried out directly using, e.g., Monte Carlo (MC) analysis to obtain the desired results. However, uncertainties in the input parameters of LCA models are often not well known. It is seldom feasible, nor necessary, to find detailed uncertainty specifications for all variable model parameters in an LCA study.

First, a "sensitivity analysis" is carried out. The term sensitivity analysis is interpreted in different ways, but we will follow the definition by Saltelli et al. (2000) who explains it as the contribution of the uncertainty in individual input parameters to the predicted model result. Uncertainties in input parameters will be quantified roughly in the sensitivity analysis. Quite often, it turns out that only a few parameters determine the uncertainty of the model 
result. For those parameters, more detailed information should then be gathered in a sequential step.

In the second step, the actual "uncertainty analysis" is carried out with the updated uncertainty information. After the uncertainty analysis, it has to be checked whether the parameters that were most important in the sensitivity analysis and for which we now have collected updated information are still the most important parameters in the uncertainty analysis. If a completely different set of parameters would come out as being most importantwhich means that our initial estimate of uncertainty in the input parameters was wrong-the analysis should be reiterated. A similar approach was also adopted by (Huijbregts et al. 2003).

\subsection{Sensitivity analysis}

The Fourier amplitude sensitivity test (FAST; Saltelli et al. 2000, 2004) has been used for the sensitivity analysis. The FAST method can calculate sensitivity indices for individual parameters but also for groups of parameters The sensitivity indices as calculated by the FAST method may be used quantitatively. The FAST method uses uncertainty distributions as input and can take into account correlation coefficients. It is a global sensitivity analysis tool, i.e., the input parameters are varied simultaneously over their full range. Simlab was the software used for the sensitivity analysis (Simlab 2004). More details with regard to the sensitivity analysis can be found in the "Electronic supplementary material."

\subsection{Refined uncertainty specification in the comparison situations}

Having determined the most sensitive parameters in the sensitivity analysis, the refined data-gathering procedure was concentrated on specifying in more detail the uncertainty for these parameters by:

- Collecting specific information about uncertainty for most influential parameters

- Assessing the suitability of the distribution function (although fitting distribution functions was often impossible due to limited data availability)

- Assessing possible existence of correlation coefficients between the most influential parameters

\subsection{Uncertainty analysis}

After updating the uncertainty information for the most influential parameters, the actual uncertainty analysis were carried out with $\mathrm{MC}$ analysis using Latin hypercube sampling. Between 5,000 and 10,000 runs were made for each MC analysis, depending on the number of variable input parameters in each comparison situation. It has been checked using the FAST method whether the most influential parameters were the parameters for which detailed uncertainty information was gathered. For the uncertainty analysis, the software package Simlab has also been used.

\subsection{Subjective versus analytical approach}

In the previous method description, we made a major assumption by disregarding the uncertainty in parameters of common background processes when comparing the CFs of the compact powder and ultraliquid product. This was defined earlier as the subjective approach. To test this assumption, the subjective and analytical approaches were compared for the IPC comparison case with MC analysis using Latin hypercube sampling.

For the subjective approach, 10,000 samples were generated for the input parameters of the compact powder and ultraliquid product independently from each other. Samples were only generated for the input parameters of processes that are different for the two LCA systems. The input parameter values of the common processes remained fixed at their default.

In the analytical approach, 10,000 samples were generated for all the input parameters of the compact powder and ultraliquid product, including the input parameters describing the common processes. However, the input parameters for the common processes of compact powder and ultraliquid were assumed to be fully correlated. A Matlab routine was developed to make this possible.

\section{Results}

The main results of the uncertainty analysis are given in Fig. 1 as histograms of the MC runs. The histograms show the differences between the calculated $\mathrm{CF}$ of compact powder minus ultraliquid for comparison situations 1,2 , and 3 calculated as $\Delta \mathrm{CF}=\mathrm{CF}_{\text {compact powder }}-\mathrm{CF}_{\text {ultraliquid. }}$.

This representation is used because it focuses on the uncertainty in the difference between the CF of the two products and not on the uncertainty in the $\mathrm{CF}$ of the individual detergents as absolute values. It is primarily in the comparison of the products that the calculated uncertainty for the individual products becomes meaningful.

Results in Fig. 1 and Table 2 indicate that the uncertainties are small with respect to the difference in mean $\mathrm{CF}$ of both detergents $\left(73 \mathrm{~g} \mathrm{CO}_{2}\right.$ eq per wash for $\triangle \mathrm{CF}$ ) in comparison situation 1 . The uncertainties are so small that the CF of compact powder is always higher than the $\mathrm{CF}$ of the ultraliquid product. Clearly, the detergent 


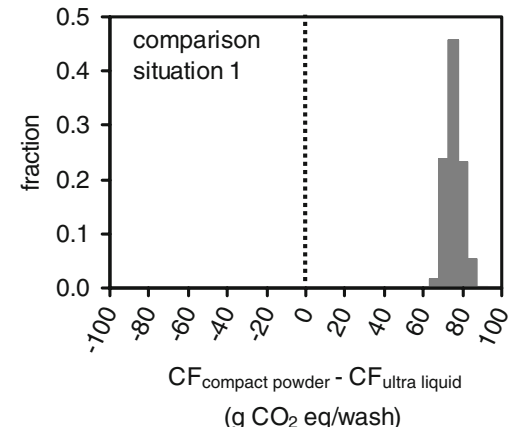

( $\mathrm{g} \mathrm{CO}_{2}$ eq/wash)

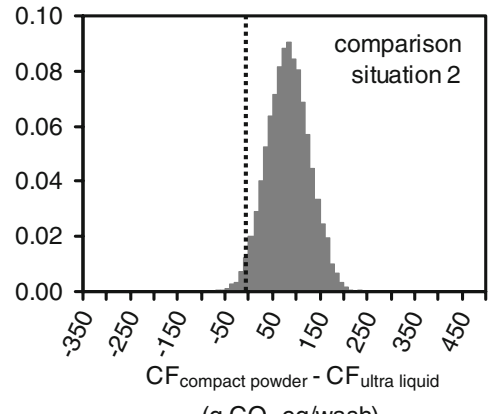

( $\mathrm{g} \mathrm{CO}_{2}$ eq/wash)

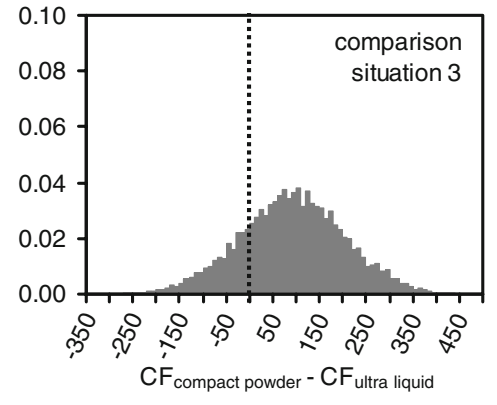

( $\mathrm{g} \mathrm{CO}_{2}$ eq/wash)

Fig. 1 Histograms showing the differences between the calculated CF of compact powder and ultra liquid for comparison situations 1, 2, and 3 . The dotted line indicates the point where $\mathrm{CF}_{\text {compact powder }}=\mathrm{CF}_{\mathrm{ultraliquid}}$. Notice the different scale for comparison situation 1

formula with the smallest absolute CF of the two is the ultraliquid.

For comparison situation 2, the analysis still suggests that the ultraliquid product is the product that tends to have the lowest $\mathrm{CF}$. Figure 1 shows that there are only a few $\mathrm{MC}$ runs where the $\mathrm{CF}$ of the compact powder product is lower than that of the ultraliquid. Or, in other words, due to the fact that some different choices could have been made by independent LCA practitioners, it might be that an inverse conclusion is reached. Table 2 indicates that such could happen in about $5 \%$ of the cases.

Under comparison situation 3, the ultraliquid cannot reliably be identified as the product with the smallest CF. Figure 1 shows that a significant fraction (in $23 \%$ of the cases, see Table 2) of the CF values calculated in the MC analysis for the compact powder product are smaller than those of ultraliquid. Hence, in this case, a product selection based on CF is largely blurred by uncertainty.

Figure 2 shows which parameter groups had the largest contribution to the uncertainty in the $\mathrm{CF}$ of compact powder or ultraliquid product under comparison situations 1, 2, and 3. The group labels in Fig. 2 are explained in Table 1. In comparison situation 1, the uncertainty in the $\mathrm{CF}$ is dominated by the detergent manufacturing process and uncertainties in the amount of transport needed. In comparison situation 2, the overall CF uncertainty is dominated by uncertainties in chemical production, use phase (efficiency of washing machine), and GHGs emanating from wastewater treatment. Similarly, in comparison situation 3, washing conditions, chemical production, and wastewater treatment dominate the total uncertainty of the calculated CF.

The parameters that dominate uncertainty are the parameters already identified in the sensitivity analysis as being the most influential and for which more detailed uncertainty information was gathered before the uncertainty analysis. Therefore, no further iteration according the stepwise approach was necessary.

\section{Discussion}

From a methodological standpoint, several key assumptions or simplifications have been made in this study. First, an important simplification, which particularly impacts the analysis of uncertainties in comparison situation 1 , is that the uncertainties in common background processes can be neglected (the subjective approach). The analytical approach, however, is likely more powerful but requires more effort on the part of the LCA practitioner. To further aid development of uncertainty methods for LCA and CF calculations, the results of the subjective approach and
Table 2 Statistical properties of the distribution functions that describe the uncertainty of the difference between the CF of the Compact Powder and Ultra Liquid product, as shown a histogram in Fig. 1. The deterministic value for the calculated $\triangle \mathrm{CF}$ is given on the first row

\begin{tabular}{llccc}
\hline Parameter & Unit & $\begin{array}{l}\text { Comparison } \\
\text { situation } 1\end{array}$ & $\begin{array}{l}\text { Comparison } \\
\text { situation 2 }\end{array}$ & $\begin{array}{c}\text { Comparison } \\
\text { situation 3 }\end{array}$ \\
\hline$\Delta$ CF deterministic & $\mathrm{g} \mathrm{CO}_{2}$ eq/wash & 73 & 73 & 82 \\
Mean & $\mathrm{g} \mathrm{CO}_{2}$ eq/wash & 73 & 73 & 81 \\
$\mathrm{SD}$ & $\mathrm{g} \mathrm{CO}_{2}$ eq/wash & 4 & 44 & 114 \\
10th percentile & $\mathrm{g} \mathrm{CO}_{2}$ eq/wash & 68 & 16 & -63 \\
90th percentile & $\mathrm{g} \mathrm{CO}_{2}$ eq/wash & 78 & 130 & 226 \\
Min & $\mathrm{g} \mathrm{CO}_{2}$ eq/wash & 61 & -75 & -340 \\
Max & $\mathrm{g} \mathrm{CO}_{2}$ eq/wash & 89 & 226 & 483 \\
$\mathrm{CF} \mathrm{ul>CF} \mathrm{cp}$ & $\%$ & 0 & 5 & 23 \\
\hline
\end{tabular}



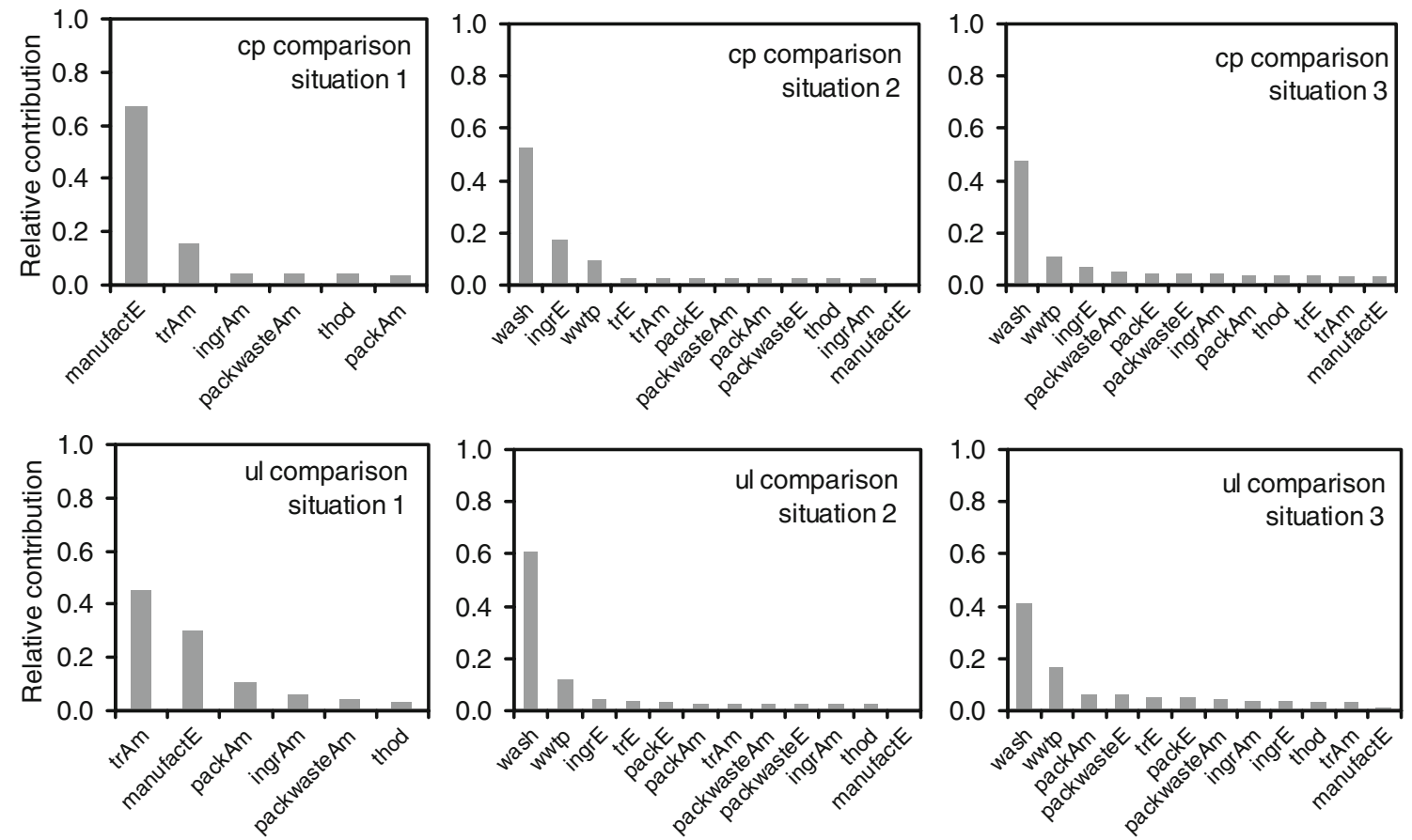

Fig. 2 Relative contribution of the different parameter groups to total uncertainty of the CF according to FAST total indices for compact powder $(c p)$ and ultraliquid $(u l)$ in comparison situations 1,2 , and 3 after refinement of the input parameters, i.e., in the uncertainty analysis

analytical approach were compared for comparison situation 1 (Fig. 3). The two histograms show the $\Delta \mathrm{CF}$ as calculated in the subjective and analytical approach. Since the two histograms in Fig. 3 are very similar, the subjective approach to simply neglect uncertainties in the common background processes is justified and is a suitable way to handle uncertainties in the comparison of products. However, it should not be forgotten that having a similar background system in two LCA systems increases the precision of the comparative result; it does not increase its accuracy. Notice that the uncertainties in Fig. 3 for the subjective approach differ from those in Fig. 1 because the

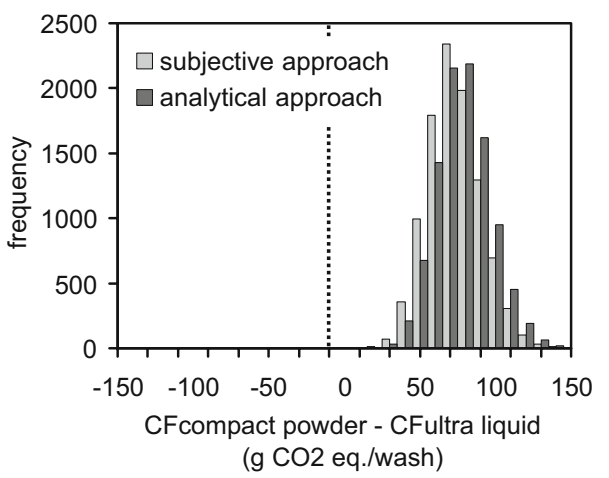

Fig. 3 Histogram showing the differences between the calculated CFs of compact powder and ultraliquid for the subjective and analytical comparison approach, applied to comparison situation 1. The $y$-axis shows the number of observations. The dotted line indicates the point where $\mathrm{CF}_{\text {compact powder }}=\mathrm{CF}_{\text {ultraliquid }}$ uncertainty in the input parameters has been specified differently.

A second key assumption in our analysis of uncertainties in the three comparison situations is to ignore correlation coefficients between the input parameters, besides the full correlation between the input parameters in comparison situation 1 that has been taken into account with the subjective approach. No quantitative information could be found for the specification of correlation coefficients between input parameters. Generally speaking, correlation coefficients between input parameters can potentially influence the uncertainty in the response variable of models if they are large, i.e., in the order of 0.7 or higher (Janssen et al. 1990). The influence of correlation coefficients in LCA models is largely unexplored (Lloyd and Ries 2007). For comparison situation 1, the input parameter that has a large contribution to the uncertainty of the $\mathrm{CF}$ is the detergent manufacturing process. One may expect that the $\mathrm{CF}$ of compact powder and ultraliquid manufacturing is strongly correlated if they use the same sources of energy (e.g., they draw electricity from the same grid if produced in the same factory or country). If indeed the CF of powder and liquid manufacturing processes is strongly correlated, the resulting uncertainties when comparing both products will be even smaller than in the current calculations. This would only strengthen the conclusions of the analysis.

In comparison situations 2 and 3 , the influence of correlation coefficients between the input parameters is likely to be small. The input parameters that have the 
largest contribution to the $\mathrm{CF}$ uncertainty, i.e., the input parameters describing the use phase, are unlikely to be strongly correlated.

This study compares the uncertainty levels of the CFs for two detergent types, under different but realistic viewpoints. It shows by means of a detergent example that $\mathrm{CFs}$ of products, if calculated by independent parties and only based on the ISO guidelines, are easily blurred by uncertainty. A large absolute difference in CF would typically be needed to discriminate products on this basis. If industrial sectors are able to agree on a "code of practice" (e.g., PCR) and fix the main sources of uncertainty to default values, the picture could become more clear and informative. Main sources of uncertainty that should be addressed in a PCR according to our sensitivity analysis results would be washing temperature, energy use of the washing machine, and the GHG emissions associated with the production of electricity for the washing machine. Also, prescribing the inclusion of the end-of-life stages for the detergents and packaging material would in total reduce uncertainty about $70 \%$ (see Fig. 2).

\section{Conclusions}

The reported estimates of uncertainties for the CFs of detergents illustrate the order of magnitude of the uncertainties in the different comparison situations, but it should be borne in mind that this is likely a favorable scenario for product CF labels since many other disposable consumer product categories are less studied. CF calculations for products can only provide a fair comparison if the LCA background system used for the two products is the same and exactly the same choices in the foreground system are made. If the $\mathrm{CL}$ schemes are to be implemented for detergent products, this study gives directions on how to make the CF label as meaningful as possible. Furthermore, the stepwise analysis and the subjective approach greatly simplified the uncertainty analysis in this case study.

\section{Recommendations and outlook}

Uncertainty analysis is an indispensable tool if the results of different LCA/CF models are to be closely compared. Currently, data collection and the analyses require a lot of effort from the LCA practitioner. Yet, due to the significance of decisions based on the LCA/CF results, it is recommended that sensitivity and uncertainty analyses be conducted and the ranges of uncertainty be given together with the single (mean) values. This should become common practice and an integral part of future international CF specifications and standards. Simplification of uncertainty analysis for LCA will be needed for a wider adoption of this requirement.

The results of this study illustrate that, due to the magnitude of uncertainty in the numbers and without the agreement on PCRs within product sectors or categories, the results of carbon labeling schemes are unlikely to provide precise and meaningful information to the consumer. The focus of PCRs in the detergent category should focus on the washing phase and end-of-life stages of detergents and packaging material.

Open Access This article is distributed under the terms of the Creative Commons Attribution Noncommercial License which permits any noncommercial use, distribution, and reproduction in any medium, provided the original author(s) and source are credited.

\section{References}

APME (1996) Information system on plastic waste management in Western Europe; European overview; 1994 data. Brussels, Belgium

Boustead I, Fawer M (1998) Ecoprofiles of perborates. CEFIC, Brussels BSI (2008) PAS 2050-specification for the assessment of the life cycle greenhouse gas emissions of goods and services. British Standards Institute, London

BUWAL (1996) Okoinventare für Verpackungen. Band 2, Schriftenreihe umwelt n. 250/II. Bundesambt für Umwelt, Wald und Landschaft, Bern

Ecobilan (2008) Waste management life cycle assessment software tool. Demo version available at http://www.ecobalance.com/ uk wisard.php

Efron B, Tibshirani R (1991) Statistical data analysis in the computer age. Science 253(5018):390-395

EMPA (1999) Life cycle inventories for the production of detergent ingredients. Report no. 244. Swiss Federal Laboratories for Materials Testing and Research (EMPA), St. Gallen, Switzerland, ISBN 3-905594-09-9

ETH (1996) Ökoinventare für Energiesysteme (Teil I-VII). ETH, Zürich

Franke M, Klüppel H, Kirchert K, Olschewski P (1995) Oekobilanzierung-Sachbilanz für die Waschmttel-Konfektionierung. Tenside Surfact Det 32:508-514

Frischknecht R, Jungbluth N, Althaus H-J, Doka G, Heck T, Hellweg S, Hischier R, Nemecek T, Rebitzer G, Spielmann M, Wernet G (2007) Overview and methodology. Ecoinvent report no.1, Swiss Centre for Life Cycle Inventories, Duebendorf

Guinée JB, Heijungs R (2007) Calculating the influence of allocation scenarios in fossil fuel chains. Int J LCA 12(3):173-180

Heijungs R, Huijbregts MAJ (2004) A review of approaches to treat uncertainties in LCA. In: Pahl-Wostl C, Schmidt S, Rizzoli AE, Jakeman AJ (eds) Complexity and integrated resources management. Transactions of the 2nd Biennial Meeting of the International Environmental Modelling and Software Society, vol. 1, pp 332-339. iEMSs, June 2004

Houghton JT, Ding Y, Griggs DJ, Noguer M, Van der Linden PJ, Xiaosu D (2001) IPCC Third Assessment Report: climate change 2001: the scientific basis. Cambridge University Press, Cambridge

Huijbregts MAJ, Giljamse W, Ragas MJ, Reijnders L (2003) Evaluating uncertainty in environmental life-cycle assessment. A case study comparing two insulation options for a Dutch onefamily dwelling. Environ Sci Technol 37(11):2600-2608 
ISO (2000) Environmental labels and declarations-Type III environmental declarations (ISO 14025:2000). ISO, Geneva

ISO (2006a) Environmental management-life cycle assessmentprinciples and framework (ISO 14040:2006). ISO, Geneva

ISO (2006b) Environmental management-life cycle assessmentrequirements and guidelines (ISO 14044:2006). ISO, Geneva

ISO (2007) Greenhouse gases-requirements for greenhouse gas validation and verification bodies for use in accreditation or other forms of recognition (ISO 14065:2007). ISO, Geneva

Janssen PHM, Slob W, Rotmans J (1990) Gevoeligheidsanalyse en onzekerheidsanalyse; een inventarisatie van ideëen en methoden uit de literatuur. RIVM report no 958805001. National Institute of Public Health and Environmental Protection, Bilthoven

Johnson E (2008) Disagreement over carbon footprints: a comparison of electric and LPG forklifts. Energ Policy 36(4):1569-1573

Lloyd SM, Ries R (2007) Characterizing, propagating, and analyzing uncertainty in life-cycle assessment; a survey of quantitative approaches. J Ind Ecol 11(1):161-175

Matthews HS, Hendrickson CT, Weber CL (2008) The importance of carbon footprint estimation boundaries. Environ Sci Technol 16 (42):5839-5842

NEF (2008) Energy efficient washing machines. National Energy Foundation. http://www.nef.org.uk/energysaving/labels.htm. Accessed April 2008

Pant R, Köhler A, De Beaufort A, Braune A, Frankl P, Hauschild M, Klöpffer W, Kreissig J, Linfors LG, Masoni P, Pennington D, Riise E (2008) Standardisation efforts to measure greenhouse gases and 'carbon footprinting' for products. Int J Life Cycle Assess 13(2):87-88

Saltelli A, Tarantola S, Campolongo F (2000) Sensitivity analysis as an ingredient of modeling. Stat Sci 15(4):377-397

Saltelli A, Tarantola S, Campolongo F, Ratto M (2004) Sensitivity analysis in practice; a guide to assessing scientific models. Wiley, New York. ISBN 978-0470-87093-8

Saouter E, Van Hoof A (2001) A database for life-cycle assessment of Procter \& Gamble laundry detergents. Int J Life Cycle Assess 7 (2): $103-114$

Simlab (2004) Version 2.2 Simulation environment for uncertainty and sensitivity analysis, developed by the Joint Research Centre of the European Commission

Staatsblad (2006) Besluit van 20 oktober 2006, houdende regels met betrekking tot het gebruik van biobrandstoffen in het wegverkeer

UNEP (2007) Global Environment Outlook (GEO-4). ISBN 978-92807-2836-1

US-EPA (1989) Exposure factors handbook. EPA, Washington

US FTC (1998) US Federal Trade Commission Guides for the Use of Environmental Marketing Claims, in accordance with section 1501.3 of the Council on Environmental Quality's regulations for implementing procedures of the National Environmental Policy Act, 42 U.S.C 4321

WBSCD/WRI (2007) The greenhouse gas protocol. World Business Council for Sustainable Development and World Resources Institute, Geneva 